\title{
A Back-Stepping Sliding Mode Control Strategy Based on Extended Observer for Photovoltaic Grid-Connected Inverter
}

\author{
Hui Ren, Jisheng $\mathrm{Li}^{*}$, Dan Li, Guodong You and Zhaojing Wang \\ College of Electronic Information and Automation, Tianjin University of Science and Technology, Hexi District, Tianjin \\ 300222, China \\ ${ }^{*}$ Corresponding author
}

\begin{abstract}
In the photovoltaic power generation system, the grid-connected inverter is an important link to realize system connection with the electricity grid, but the existence of various interferences of the external environment and the system itself brings a series of problems. In this paper, the circuit of the LCL is used to filter of photovoltaic grid-connected inverter output, and step-sliding mode control and the extended state observer are combined to complete the tracking control of the inverter system. Three state variables of the control strategy are the output voltage of inverter system its first and second derivative, back-stepping and sliding mode control are combined, and the extended state observer is designed to estimate the composite interference, then its output compensates to the back-stepping sliding mode controller. The effectiveness of the control strategy is proved by the simulation of photovoltaic grid-connected inverter system in Matlab.
\end{abstract}

Keywords-grid-connected inverter system; LCL filter circuit; back-stepping sliding mode control; extended state observer

\section{INTRODUCTION}

With the deterioration of environmental issues, the grid connected photovoltaic power generation technology has been paid attention to by many scholars to promote the strategy of sustainable development. PV grid-connected inverter system is the key to the realization of power supply grid in photovoltaic power generation system. Therefore, the establishment of mathematical model and control technology have become one of the main directions of domestic and foreign research .

The photovoltaic grid-connected inverter system in this paper adopts LCL output filter circuit, and combines the back-step sliding mode control and the extended state observer to complete the tracking control of the inverter system. Firstly, the continuous mathematical model of the inverter system is established by using the state space averaging method. Taking the output voltage of the inverter system and its first order and second derivative as the state variables, the back-stepping method and the sliding mode control are combined to establish the photovoltaic Back-stepping Sliding Mode Control Model for Grid - Connected Inverter System. And then combine the uncertainties caused by the internal and external factors in the system into a composite interference, and then use the extended state observer to estimate the interference and output it to the back stepping sliding mode controller. Therefore, the control strategy is not the upper bound information of known composite interference needs to be realized, and the buffeting phenomenon of the system can be effectively weakened while realizing the system control.

II. ESTABLiSHMENT OF MATHEMATICAL MODEL FOR SingLE - Phase Photovoltaic Grid - CONNECTEd INVERTER System

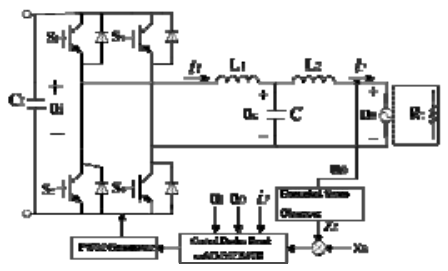

FIGURE I. SINGLE-PHASE PHOTOVOLTAIC GRID-CONNECTED INVERTER SYSTEM MAIN CIRCUIT DIAGRAM

The single-phase photovoltaic grid-connected inverter system includes four switching tubes $S_{1}-S_{4}$ and a filter circuit composed of $L_{1}, L_{2}$ and $C$. The currents of the inductors $L_{1}$ and $L_{2}$ are $i_{1}$ and $i_{2}$, the voltage of the capacitor $C$ is $u_{c}$, the grid voltage is the $u_{0}$, the PV side of the output DC voltage $u_{i}$, in order to facilitate the calculation of the grid circuit equivalent to a reactor $R_{L}$.

Figure 1 in the inverter system DC side voltage for the photovoltaic bus voltage $u_{i}$, by controlling the four switch tube to achieve DC / AC function.Assuming that the switch in the inverter system is ideal, the state equation $\left[x_{1}, x_{2}, x_{3}\right]=\left[u_{0}, \dot{u}_{0}\right.$, $\left.\ddot{u}_{0}\right]$ is defined by the method in [1,2] to obtain the state equation of the photovoltaic grid-connected inverter system state:

$$
\begin{gathered}
\left\{\begin{array}{l}
\dot{\boldsymbol{x}}=\boldsymbol{A} \boldsymbol{x}+\boldsymbol{B u}+\boldsymbol{D} \\
\boldsymbol{y}=\boldsymbol{C} \boldsymbol{x}
\end{array}\right. \\
\boldsymbol{x}=\left[\begin{array}{lll}
x_{1} & x_{2} & x_{3}
\end{array}\right]^{\mathrm{T}} ; \boldsymbol{u}=\left[u_{i}\right] ; \boldsymbol{A}=\left[\begin{array}{ccc}
0 & 1 & 0 \\
0 & 0 & 1 \\
a_{1} & a_{2} & a_{3}
\end{array}\right]
\end{gathered}
$$




$$
\begin{gathered}
\boldsymbol{B}=\left[\begin{array}{l}
0 \\
0 \\
b
\end{array}\right] ; \mathbf{C}=\left[\begin{array}{lll}
1 & 0 & 0
\end{array}\right] ; \mathbf{D}=\left[\begin{array}{l}
0 \\
0 \\
h
\end{array}\right] ; \\
a_{1}=-\frac{\mathrm{R}_{\mathrm{L}}}{\mathrm{L}_{1} \mathrm{~L}_{2} \mathrm{C}} ; a_{2}=-\frac{\mathrm{L}_{1}+\mathrm{L}_{2}}{\mathrm{~L}_{1} \mathrm{~L}_{2} \mathrm{C}} ; \quad a_{3}=-\frac{\mathrm{R}_{\mathrm{L}}}{\mathrm{L}_{2}} ; \\
b=\frac{\mathrm{R}_{\mathrm{L}}}{\mathrm{L}_{1} \mathrm{~L}_{2} \mathrm{C}}(2 N-1) ; h=\beta_{1} x_{1}+\beta_{2} x_{2}+\beta_{3} x_{3}+g ;
\end{gathered}
$$

Where: $u$ is the system input; $y$ is the system output; $N$ is the duty cycle of the switch $\mathrm{S}_{1}, \mathrm{~S}_{4}, \mathrm{~N} \in[0,1] ; \quad \beta_{1}, \beta_{2}, \beta_{3}$ for the uncertain factors caused by the system inductance and capacitance, $g$ for the interference caused by the error, $h$ for the composite interference.

\section{CONTROL StRATEgy OF PhOtovoltaic GRID - CONNECTED INVERTER SYSTEM}

Because the mathematical model (1) of the PV grid-connected inverter system is a third-order system, if the design method given in [3] is used directly, it is necessary to design a fourth-order extended state observer, But a large number of theoretical analysis and simulation results show that, when the order of the extended observer exceeds the third order, it is difficult to find the appropriate design parameters to ensure that the states of the designed extended state observer converge to the respective states in the system[4-6]. In the photovoltaic inverter system (1), since the state variables $\mathrm{x} 1$ and $\mathrm{x} 2$ can be measured directly, it is only necessary to observe the composite interference $h$ which can not be directly measured and the corresponding state $\mathrm{x} 3$. According to the method of compound interference in [7], we first design the sliding mode surface, and then design the second order extended state observer to estimate the switching function and the composite interference. Based on this design, the expansion of the state observer needs to adjust the design parameters less,with a simple structure, easy to achieve the advantages[8].

\section{A. Construction Of Back-stepping Sliding Mode Controller}

Let the expected output of the PV inverter system be $x_{d}$ and assume that the first and third derivatives of the desired output are present and bounded. Design the controller to make the closed-loop system stable, the output y can achieve the desired output $\mathrm{x}_{\mathrm{d}}$ tracking.

First define the error variable:

$$
\left\{\begin{array}{l}
\omega_{1}=y-x_{d}=x_{1}-x_{d} \\
\omega_{2}=x_{2}-\gamma_{1} \\
\omega_{3}=x_{3}-\gamma_{2}
\end{array}\right.
$$

Where $\omega_{1}$ is the tracking error variable, $\gamma_{1}, \gamma_{2}$ is the virtual control of the subsystems $\omega_{1}, \omega_{2}$.

The back-stepping system decomposes the nonlinear system that satisfies the parameter strictly feedback into subsystems that do not exceed the order of the system, and designs the Lyapunov function and the intermediate virtual control volume for each subsystem until it is "retreated" to the whole system[9] . Because the PV grid-connected inverter system (1) is a third-order system, the system back-step design can be divided into three steps:

The first step is to select the Lyapunov function $Y_{1}$ as:

$$
\mathrm{Y}_{1}=\frac{1}{2} \omega_{1}^{2}
$$

$$
\dot{\mathrm{Y}}_{1}=\omega_{1} \cdot \dot{\omega}_{1}=\omega_{1}\left(\dot{x}_{1}-\dot{x}_{d}\right)=\omega_{1}\left(\omega_{2}+\gamma_{1}-\dot{x}_{d}\right)
$$

Let the virtual control of the subsystem $\omega_{1}$ be $\gamma_{1}$ :

$$
\gamma_{1}=-\lambda_{1} \omega_{1}+\dot{x}_{d}\left(\lambda_{1}>0\right)
$$

Substituting equation (5) into equation (4):

$$
\mathrm{Y}_{1}=-\lambda_{1} \omega_{1}^{2}+\omega_{1} \omega_{2}
$$

The second step is to select the Lyapunov function

$$
\mathrm{Y}_{2}=\mathrm{Y}_{1}+\frac{1}{2} \omega_{2}^{2}
$$

Derived for $\mathrm{Y}_{2}$ :

$$
\dot{\mathrm{Y}}_{2}=\dot{\mathrm{Y}}_{1}+\omega_{2} \dot{\omega}_{2}=-\lambda_{1} \omega_{1}^{2}+\omega_{1} \omega_{2}+\omega_{2}\left(\omega_{3}+\gamma_{2}-\dot{\gamma}_{1}\right)
$$

Let the virtual control of the subsystem $\omega_{2}$ be $\gamma_{2}$ :

$$
\gamma_{2}=-\lambda_{2} \omega_{2}-\omega_{1}+\dot{\gamma}_{1}\left(\lambda_{2}>0\right)
$$

Substituting equation (9) into equation (8):

$$
\dot{\mathrm{Y}}_{2}=-\lambda_{1} \omega_{1}^{2}-\lambda_{2} \omega_{2}^{2}+\omega_{2} \omega_{3}
$$

The third step defines the switching function as:

$$
s=\omega_{3}
$$

The sliding mode approximation law can be expressed

as:

$$
\dot{s}=-k s-l \operatorname{sgn}(s) \quad(k>0, l>0)
$$

Where $\mathrm{k}$ and 1 determine the convergence speed of the slip surface, which can be seen by Eq. (12),the larger the $k$ and $l$, the faster the convergence rate of the system. However, when $\mathrm{k}$ and 1 become larger, the control input amplitude also becomes larger. Therefore, in the exponential approximation 
law, it is possible to increase $\mathrm{k}$ and decrease the time to achieve fast convergence and weaken the buffeting [10].

In the formula (11), we derive $s$ :

$$
\begin{aligned}
& \dot{s}=\dot{\omega}_{3}=\dot{x}_{3}-\dot{\gamma}_{2}=\frac{\mathrm{R}_{\mathrm{L}}}{\mathrm{L}_{1} \mathrm{~L}_{2} \mathrm{C}}(2 D-1) u_{i}-\frac{\mathrm{R}_{\mathrm{L}}}{\mathrm{L}_{1} \mathrm{~L}_{2} \mathrm{C}} x_{1} \\
& -\frac{\mathrm{L}_{1}+\mathrm{L}_{2}}{\mathrm{~L}_{1} \mathrm{~L}_{2} \mathrm{C}} x_{2}-\frac{\mathrm{R}_{\mathrm{L}}}{\mathrm{L}_{2}} x_{3}-\dot{\gamma}_{2}+h \\
& f\left(x_{1}, x_{2}, x_{3}\right)=-\frac{\mathrm{R}_{\mathrm{L}}}{\mathrm{L}_{1} \mathrm{~L}_{2} \mathrm{C}} x_{1}-\frac{\mathrm{L}_{1}+\mathrm{L}_{2}}{\mathrm{~L}_{1} \mathrm{~L}_{2} \mathrm{C}} x_{2}-\frac{\mathrm{R}_{\mathrm{L}}}{\mathrm{L}_{2}} x_{3} \\
& \dot{s}=\dot{\omega}_{3}=\dot{x}_{3}-\dot{\gamma}_{2}=\frac{\mathrm{R}_{\mathrm{L}}}{\mathrm{L}_{1} \mathrm{~L}_{2} \mathrm{C}}(2 D-1) u_{i}+ \\
& f\left(x_{1}, x_{2}, x_{3}\right)-\dot{\gamma}_{2}+h
\end{aligned}
$$

According to (12), (13) and (15) can be designed for the controller:

$$
D=\frac{1}{2}\left[1+\frac{L_{1} L_{2} C}{R_{L} U_{i}}\left(-k s-l s y n(s)-f\left(x_{1}, x_{2}, x_{3}\right)+\dot{\gamma}_{2}-h\right)\right]
$$

As can be seen from the actual project, the controller (16) does not appear strange phenomenon. However, the presence of composite interference $h$ makes the controller unusable. In order to solve this problem, the composite interference will be estimated by designing the extended state observer and the output will be compensated to the back-step sliding mode controller[11].

\section{B. Design Of Extended State Observer}

First, the composite interference $h$ is expanded to the state $H$, then the equation (15) can be expanded to:

$$
\left\{\begin{array}{l}
\dot{s}=\frac{\mathrm{R}_{\mathrm{L}}}{\mathrm{L}_{1} \mathrm{~L}_{2} \mathrm{C}}(2 D-1) u_{i}+f\left(x_{1}, x_{2}, x_{3}\right)-\dot{\gamma}_{2}+H \\
\dot{H}=\phi(t)
\end{array}\right.
$$

Where $\phi(t)$ is the differential of the composite interference $\mathrm{h}$.

For the above-mentioned second-order expansion state observer can be designed as:

$$
\left\{\begin{array}{l}
E_{1}=Z_{1}-s \\
\dot{Z}_{1}=Z_{2}+\frac{\mathrm{R}_{\mathrm{L}}}{\mathrm{L}_{1} \mathrm{~L}_{2} \mathrm{C}}(2 D-1) u_{i}+f\left(x_{1}, x_{2}, x_{3}\right)-\dot{\gamma}_{2}-\eta_{1} E_{1} \\
\dot{Z}_{2}=-\eta_{2} f a l\left(E_{1}, \tau, \varepsilon\right)
\end{array}\right.
$$

Where $E_{1}$ is the estimated error, $Z_{1}$ and $Z_{2}$ are the observer output, $\eta_{1}>0, \eta_{2}>0$ the function $\operatorname{fal}\left(E_{1}, \tau, \varepsilon\right)$ is defined as:

$$
f a l\left(E_{1}, \tau, \varepsilon\right)= \begin{cases}\left|E_{1}\right|^{\tau} \operatorname{sgn}\left(E_{1}\right), & \left|E_{1}\right|>\varepsilon \\ \frac{E_{1}}{\varepsilon^{1-\tau}}, & \left|E_{1}\right| \leq \varepsilon\end{cases}
$$

Where $0<\tau<1, \varepsilon>0$.

For the extended state observer (18), we have the following conclusions:

Lemma 1: For the expansion state observer (18), there are appropriate design parameters $\eta_{1}, \eta_{2}, \tau, \varepsilon$ such that the output $Z_{1}$ and $Z_{2}$ of the observer converge to a neighborhood of $s$ and $h$, respectively.

Proof: First, the estimated error $E_{2}=Z_{2}-h$ is defined, then there is a suitable $\eta_{1}, \eta_{2}$ makes the observer stable, and the stability of the extended state observer is shown in literature[12].

The estimated error equation of the observer is:

$$
\left\{\begin{array}{l}
\dot{E}_{1}=E_{2}-\eta_{1} E_{1} \\
\dot{E}_{2}=-\phi(t)-\eta_{2} \operatorname{fal}\left(E_{1}, \tau, \varepsilon\right)
\end{array}\right.
$$

When the observer is stable, there are:

$$
\left\{\begin{array}{l}
E_{2}-\eta_{1} E_{1}=0 \\
-\phi(t)-\eta_{2} \operatorname{fal}\left(E_{1}, \tau, \varepsilon\right)=0
\end{array}\right.
$$

The estimated error at this time is:

$$
\left\{\begin{array}{l}
E_{1}=-f a l^{-1}\left(\phi(t) / \eta_{2}\right) \\
E_{2}=\eta_{1} E_{1}=-\eta_{1} f a l^{-1}\left(\phi(t) / \eta_{2}\right)
\end{array}\right.
$$

Combined with (19), when $\left|E_{1}\right|>\varepsilon$, the estimated error is:

$$
\left\{\begin{array}{l}
\left|E_{1}\right|=\left|\phi(t) / \eta_{2}\right|^{1 / \tau} \\
\left|E_{2}\right|=\eta_{1}\left|\phi(t) / \eta_{2}\right|^{1 / \tau}
\end{array}\right.
$$

When $\left|E_{1}\right| \leq \varepsilon$, the estimated error is:

$$
\left\{\begin{array}{l}
\left|E_{1}\right|=\left|\phi(t) \varepsilon^{1 / \tau}\right| / \eta_{2} \\
\left|E_{2}\right|=\eta_{1}\left|\phi(t) \varepsilon^{1 / \tau}\right| / \eta_{2}
\end{array}\right.
$$


From the formula (23) and (24) we can see that the parameters $\eta_{1}, \eta_{2}, \tau, \varepsilon$ determine the size of the estimated error. In the reference [13], by selecting the appropriate design parameters, we can ensure that the estimation errors $E_{1}$ and $E_{2}$ are sufficiently small, that is, the output $Z_{1}$ and $Z_{2}$ of the observer can converge into a neighborhood of $s$ and $h$, respectively.

\section{Stability Analysis Of Closed Loop System}

The composite interference $\mathrm{h}$ is estimated by the above-mentioned extended state observer, and then the controller (16) is modified by using its output $Z_{2}$ :

$$
D=\frac{1}{2}\left[1+\frac{L_{1} L_{2} C}{R_{L} U_{i}}\left(-k s-l s y n(s)-f\left(x_{1}, x_{2}, x_{3}\right)+\dot{\gamma}_{2}-Z_{2}\right)\right]
$$

Based on the above design process, the following conclusions can be drawn.

Theorem 1 For a photovoltaic grid-connected inverter system (1) with parametric uncertainties and external disturbances, the design expansion state observer (18) estimates the composite interference $\mathrm{h}$, the design parameters $\lambda_{1}>0, \lambda_{2}>0$ and $k>0$, under the action of the controller (25), the closed-loop system will be attracted to the sliding surface and will eventually stabilize, and the system's position output can track the desired trajectory.

Prove Select Lyapunov function:

$$
\mathrm{Y}=\mathrm{Y}_{2}+\frac{1}{2} s^{2}
$$

The derivative of $Y$, in conjunction with the formula (8), (15) and a controller (25), then there will be:

$$
\begin{aligned}
\dot{Y}= & \dot{Y}_{2}+s \dot{s} \\
= & -\lambda_{1} \omega_{1}^{2}-\lambda_{2} \omega_{2}^{2}+\omega_{2} \omega_{3}+ \\
& s\left(\frac{\mathrm{R}_{\mathrm{L}}}{\mathrm{L}_{1} \mathrm{~L}_{2} \mathrm{C}}(2 D-1) u_{i}+f\left(x_{1}, x_{2}, x_{3}\right)-\dot{\gamma}_{2}+h\right) \\
= & -\lambda_{1} \omega_{1}^{2}-\lambda_{2} \omega_{2}^{2}+\omega_{2} \omega_{3}+s\left(-k s-l \operatorname{sgn}(s)-Z_{2}\right) \\
= & -\lambda_{1} \omega_{1}^{2}-\lambda_{2} \omega_{2}^{2}+\omega_{2} \omega_{3}-k s^{2}-l|s|+s\left(h-Z_{2}\right) \\
= & -\boldsymbol{E}^{\mathrm{T}} \boldsymbol{P} \boldsymbol{E}-l|s|+s\left(h-Z_{2}\right)
\end{aligned}
$$

Where $E=\left[e_{1}, e_{2}, e_{3}\right]^{\mathrm{T}}$,

$$
\mathbf{P}=\left[\begin{array}{ccc}
k_{1} & 0 & -\frac{1}{2} \\
0 & k_{2} & 0 \\
0 & -\frac{1}{2} & k
\end{array}\right]
$$

Then the order of the order $\mathrm{P}$ of the matrix $\mathrm{p}$ is as follows:
The first order of the master subordinate is: $k_{1}$;

The second order order master is:

$$
\left|\begin{array}{cc}
k_{1} & 0 \\
0 & k_{2}
\end{array}\right|=k_{1} k_{2} ;
$$

The third order order master is: $|\mathbf{P}|=k_{1} k_{2} k$;

Since the selected design parameters $k_{1}, k_{2}$ and $k$ are both greater than zero, the first and second order and the third order order prime formulas of the matrix $\mathbf{P}$ are all greater than zero, ie the matrix $\mathbf{P}$ is a positive definite matrix.

From (27) can be obtained:

$$
\dot{Y} \leq-\xi_{\text {min }}(\mathbf{P})\|\mathbf{E}\|^{2}-l|s|+s\left(h-Z_{2}\right) \leq-\xi_{\text {min }}(\mathbf{P})\|\mathbf{E}\|^{2}-\left(l-\left|E_{2}\right|\right)|s|
$$

Where $\xi_{\min }(\mathbf{P})$ is the smallest eigenvalue of the matrix P.

From theorem 1 we can know that by choosing the appropriate parameters $\eta_{1}, \eta_{2}, \tau, \varepsilon$, the estimation error will converge to a certain field of zero, so there is such a parameter $l$ such that $l>\left|E_{2}\right|$ [14].From (29) we can see that there is $\dot{Y}<0$ set, that is, to ensure that the system asymptotically stable, and by the definition of $Y \omega_{1}, \omega_{2}$, $s$ and $Y$ are bounded.

If the $M=\boldsymbol{E}^{\mathrm{T}} \boldsymbol{P E}$, then $l>\left|E_{2}\right|$, there $\dot{Y} \leq-M$, the calculus of the equation is available:

$$
\int_{0}^{t} M d t \leq Y(0)-Y(t)
$$

By $\dot{Y}<0$ and Y bounded to know $\lim _{t \rightarrow \infty} \int_{0}^{t} M d t<\infty$, so according to Barbalat theorem we can see that when $t \rightarrow \infty$, $M \rightarrow \infty$, so when $t \rightarrow \infty$, there will be $\omega_{i} \rightarrow 0, \mathrm{i}=1,2,3$, so $s \rightarrow 0$, that closed-loop system will be attracted to Sliding surface and will eventually converge, and the system output can achieve the desired trajectory tracking.

\section{Proof finished.}

In the sliding mode control, the parameter $l$ usually needs to satisfy $l>|h|$ due to the presence of the composite interference $h$.After combining with the extended state observer, you need to satisfy $l>\left|E_{2}\right|$. As can be seen from Lemma 1, by estimating the appropriate expansion state observer parameters, it is possible to ensure that the estimation error $E_{2}$ is sufficiently small. Therefore, the sliding mode control after combining with the expansion observer requires only a small amplitude of $l$, which attenuates the system's buffeting phenomenon. 


\section{Simulation AnALysis}

In order to verify the correctness and validity of the control method in this paper, a simulation model of the system is established according to the graph in Matlab / SIMULINK environment. The parameters of the filter are set as follows: $\mathrm{C}=10 \mu \mathrm{F}, \mathrm{L}_{1}=2 \mathrm{mH}, \mathrm{L}_{2}=1 \mathrm{mH}$, the grid frequency is $50 \mathrm{~Hz}$, the switching frequency is $20 \mathrm{kHz}$; the parameters of the controller are selected as $\lambda_{1}=300, \lambda_{2}=320, k=40$, $l=0.5$; the parameters of the expansion observer are selected as $\eta_{1}=4000, \eta_{2}=8000, \tau=0.05, \varepsilon=0.1 .1$ )

$A$. When the inverter system is stable, the load is pure resistance and the DC side voltage is controlled to $500 \mathrm{~V}$. Through the inverter control, so that the system output phase voltage $220 \mathrm{~V}$ AC voltage. Figure 2 for the stable operation of the inverter voltage and current waveform. It can be seen from Figure 2, the system has a good voltage waveform and current waveform, the waveform for the standard sine wave, and the output current of the effective value of 44 A.The inverter system achieves the desired effect during stable operation.

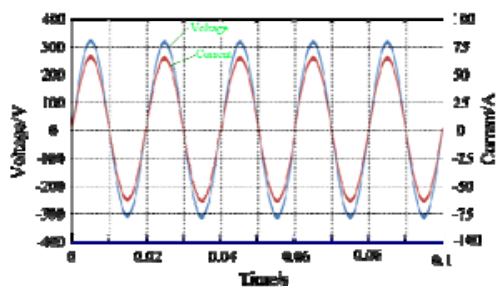

FIGURE II. STEADY RUN WHEN THE INVERTER VOLTAGE AND CURRENT WAVEFORM

$B$. Side voltage fluctuation. Figure 3 shows the DC side voltage waveform after $\mathrm{t}=0.02 \mathrm{~s}$, and Figure 4 shows the inverter voltage waveform when the DC side voltage fluctuates. It can be seen from Fig. 3 and Fig. 4 that when the DC side voltage fluctuates, although the output voltage and current fluctuate at $\mathrm{t}=0.02 \mathrm{~s}$, the interference is eliminated in one cycle, that is, after $\mathrm{t}=0.04 \mathrm{~s}$ To a steady state. It can be seen that the system has a good dynamic tracking adjustment characteristics and strong anti-jamming characteristics.

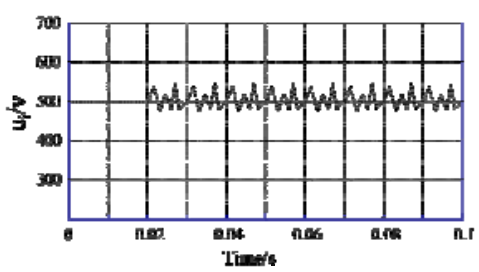

FIGURE III. WHEN THE SUDDEN INCREASE IN THE DC SIDE OF THE VOLTAGE WAVEFORM

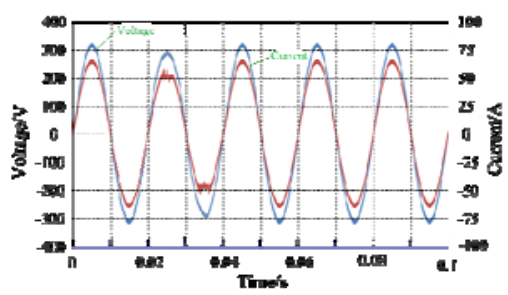

FIGURE IV. SUDDEN INTERFERENCE WHEN THE INVERTER VOLTAGE AND CURRENT WAVEFORM

$C$. When the inverter system is stable operation, the load side resistance $10 \Omega$, then the inverter system output voltage RMS $220 \mathrm{~V}$, the load side of the output current of the effective value of 44 A.Figure 5 for the sudden load resistance when the inverter voltage and current waveform. It can be seen from Fig. 5 that when $\mathrm{t}=0.02 \mathrm{~s}$, the load is suddenly increased and the load resistance is switched from the original resistor $10 \Omega$ to the resistor $20 \Omega$. At this time, the output voltage waveform is distorted, but the system is stabilized again after one cycle, And again reaches the effective value $220 \mathrm{~V}$ of the previous output voltage. At this time, the effective value of the load side current is $11 \mathrm{~A}$

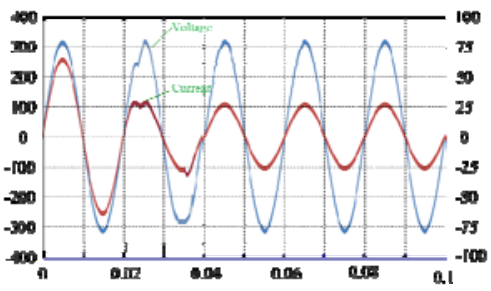

FIGURE V. WHEN THE LOAD RESISTANCE OF THE INVERTER VOLTAGE AND CURRENT WAVEFORM

D. When the inverter system is stable operation, the inverter system accesses the nonlinear load power diode. Figure 6 for the inverter system to access the nonlinear load voltage and current waveform, the voltage is sinusoidal waveform, the current is half sine wave, indicating that the system can be a good stable operation.

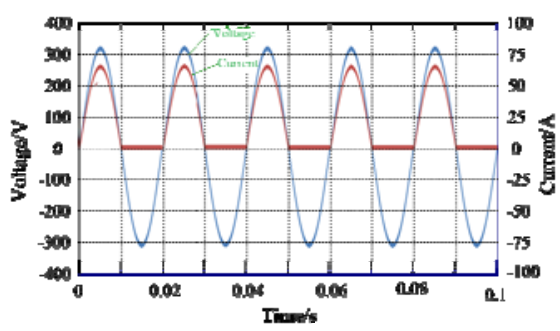

FIGURE VI. WHEN THE LOAD IS THE POWER DIODE, THE INVERTER VOLTAGE AND CURRENT WAVEFORM

\section{CONCLUSION}

Aiming at the interference problem of PV grid-connected inverter system and the of system parameter, this paper proposes a back-stepping sliding mode control strategy based on extended state observer. The composite interference is estimated by designing the extended state observer, and then 
the back-stepping sliding mode controller is designed based on the observer. Since the composite interference estimation is compensated by the expansion state observer, the upper bound of the known composite interference is not essential, so that the buffeting can be effectively reduced while realizing the system control. The simulation results show that the control strategy have good steady-state and dynamic performance. When the system is connected to a linear load or a non-linear load, the strategy can achieve effective control of the system, and the composite interference has a strong robustness.

\section{REFERENCES}

[1] Guodong YOU, Jisheng LI, Yong HOU, etal. A Back-Stepping Sliding Mode Control Strategy for Photovoltaic Grid-Connected Inverter[J]. Power Grid Technology,2015 39 (4) : 916-923.

[2] Yueming HU. Nonlinear Control Theory and Application [M]. Beijing: National Defence Industry Press,2005 : 188-193.

[3] Han J. From PID to Active Disturbance Rejection Control[J]. IEEE Transactions on Industrial Electronics. 2009, 56(3): 900-906.

[4] Ishaque K, Salam Z, Taheri H, et al, A Critical Evaluation of EA Computational Methods for Photovoltaic Cell Parameter Extraction Based on Two Diode Model [J], Solar Energy, 2011(85): 1768-1779.

[5] Ishaque K, Salam Z, Taheri H, Simple, Fast and Accurate Two-diode Model for Photovoltaic Modules [J] Solar Energy,2011(95) : 586-594.

[6] Ishaque K, Salam Z, Taheri H, et al, Modeling and Simulation of Photovoltaic System During Partial Shading Based on a Two-diode model[J].Simulation Modelling Practice and Theory,2011(19) : 1613-1626.

[7] Chen W, Ballance D J, Gawthrop P J, et al. A Nonlinear Disturbance Observer for Robotic Manipulators[J]. IEEE Transactions on Industrial Electronics. 2000, 47(4) : 932-938.

[8] Kashif I, Zainal S, Muhammad A, et al, An Improved Particle Swarm Optimization (PSO) Based MPPT for PV with Reduced Steady-state Oscillation[J].IEEE Trans on Power Electronics,2012,27(8) : 3627-3638.

[9] Yinding PENG. Research on Fuzzy Sliding Mode Variable Structure Control Strategy of Three Phase Inverter Based on Inverter System[D]. Zhengzhou: School of Electrical Engineering, Zhengzhou University,2012.

[10] Han J. Active Disturbance Rejection Control Technique-the Technique for Estimating and Compensating the Uncertainties [M]. Beijing: National Defense Industry Press, 2008.

[11] Yanwei ZHU, Xinchun SHI, Yangqing DAN, etal. Application of PSO Algorithm in Global MPPT for PV Array[J].Proceedings of the CSEE,2012,32(4) : 42-49

[12] Haitao WU, Yize SUN, Chuo MENG, Application of fuzzy Controller with Particle Swarm Optimization Algorithm to Maximum Power Point Tracking of Photovoltaic Generation system[J].Proceedings of the CSEE,2011,31(6) : 52-57.

[13] Liu F R, Duan S X, Liu F, et al, A Variable Step Size INC MPPT Method for PV Systems[J].IEEE Trans on Industrial Electronics,2008,55(7) : 2622-2628.

[14] Ishaque K, Salam Z, An Improved Modeling Method to Determine the Model Parameters of Photovoltaic Modules Using Differential Evolution (DE)[J]. Solar Energy, 2011(85): 2349-2359. 